\title{
The concept of higher order operant: A preliminary analysis
}

\author{
PAUL T. P. WONG \\ Trent University, Peterborough, Ontario, Canada
}

\begin{abstract}
Two classes of operant behavior are identified. First-order operants are reinforced by predetermined specific stimulus changes and are controlled by predetermined schedules of reinforcement. Higher order operants are capable of controlling and modifying contingencies for reinforcement, and the reinforcement for higher order operants is derived from a comparison between the initial reinforcement contingency for the first-order operant and the modified contingency. These two classes of operant behavior provide two different models of man.
\end{abstract}

It is a truism that the behavior of living organisms is affected by its consequences. According to operant analysis, a consequence capable of changing the probability of occurrence of behavior preceding it is called a reinforcement, and the behavior that is modifiable by reinforcements is called operant behavior.

Traditional operant research has focused on a subset of operant behavior, which actually encompasses all activities that are subject to change by appropriate reinforcements. This subset, according to the present analysis, is called the first order operant or simply an operant. The defining characteristics of an operant are that the reinforcement consists of a predetermined stimulus change, and that the operant is functionally related to predetermined schedules of reinforcement in a manner that rivals the regularity and precision of physical sciences. An experienced researcher in operant conditioning can look at a particular cumulative record and state with confidence what kind of contingency for reinforcement has been in effect; however, he would be at a loss trying to decide whether the cumulative record is generated by a college sophomore, a pigeon, or a rat.

What has emerged from the vast bulk of data from operant research is the picture of an organism as a passive automaton controlled by his environment: His operant behavior is essentially a servomechanistically controlled feedback loop, and the action of reinforcement is automatic and quite independent of his awareness, intent, or other higher cognitive functions.

Such a picture, while accurate, is inadequate and incomplete. It is accurate only insofar as it depicts operant behavior within the paradigm of traditional operant research, where the operant and reinforcement contingency are specified and predetermined by the experimenter, and where the conditioning chamber

The research on this manuscript was supported by Trent University President's NRC grant.

Request for reprints should be addressed to Paul T. P. Wong, Department of Psychology, Trent University, Peterborough, Ontario, K9J 7B8, Canada, Comments on this paper are welcome. The author is especially interested in communicating with individuals currently doing research related to higher order operants, or planning to pursue this line of research. greatly limits the kind of behavior that the organism can show under less restricted conditions.

Let us suppose that a rat and a scientist are in two identical Skinner boxes (which differ only in size), and they both have to press a lever to earn their keep. Let us further suppose that the contingency for reinforcement is FR 100 for both the scientist and the rat. In this situation, we can be quite confident that they will both exhibit a high rate of leverpressing and frequent postreinforcement pauses characteristic of FR responding. Even if the scientist is a rare genius, he cannot behave in a way that is different from the rat. Inside this barren chamber where only leverpressing has some effect on the otherwise totally unresponsive and unyielding environment, the scientist has no opportunity to display his brilliant intellect; there are only two alternatives open to him: copping out, which means starvation, or compliance, which means behaving like a rat. Ostensibly, he actively and adaptively operates on his environment to produce reinforcements, but in actual fact, he suffers from a severe degree of power deficiency, and is coerced to behave like a stupid rat.

But what will happen when opportunities exist for the organism to negotiate terms of reinforcement or to restructure his envronment? More specifically, what will happen when the organisms can engage in activities that can change the contingencies for reinforcement? Under these circumstances, most likely the above scientist in the Skinner box will learn to change the FR 100 schedule to a less demanding one. The operant behavior that controls and modifies contingencies for reinforcement is, according to the present analysis, called higher order operant or hi-operant.

In the case of hi-operants, the reinforcement is always derived and delayed. The reinforcing event is not a specified predetermined stimulus change, but any change in reinforcement contingency which makes it more preferable to the original contingency. It is derived because it is based on a comparison between two reinforcement contingencies: one prior to the performance of the effective hi-operant, one after. The change in environment as brought about by hi-operants is 
structural in nature. It involves a restructuring of events whereby reinforcements for the first order operant are rendered more available. The reinforcement is also delayed, because it is recognized as such only after the first order operant has been reinforced, a changed reinforcement contingency has been experienced, and a comparison between the previous and the changed reinforcement contingencies has been made. The effective hi-operant may be entirely different in topography from the first order operant. The effective hi-operant may consist of a strategy rather than a specific reponse topography.

However, not all effective hi-operants produce positive results. There are effective hi-operant which increase the gain/cost ratio, reduce the reinforcement density, and generally render the contingency for reinforcement less profitable. Such effective hi-operants are called negative hi-operants. Both positive and negative hi-operants belong to the class of operant behavior in that they are affected by their consequences, even in an indirect manner. The acquisition of hi-operants is evidenced in an increase in the probability of occurrence of positive hi-operants, but a decrease in the probability of occurrence of negative hi-operants. The result is, of course, a more profitable contingency for reinforcement. Whether hi-operants are regulated by schedules of reinforcement in the same manner as first order operants remains an empirical question, but it is clearly feasible to make positive hi-operants effective according to certain schedules, these schedules, in turn, may be modified by other hi-operants. Thus, we can conceptualize a hierarchical structure of operant behavior: a second order operant modifies the reinforcement contingency that controls a first order operant, and a third order operant modifies a second order operant, and so on. At present, only second order operant has been clearly demonstrated in college students. ${ }^{1}$

In situations where higher order operants are demonstrated, a rather different picture of man emerges. $\mathrm{He}$ is no longer a passive automaton controlled by his environment, but an active problem solver and controller of his environment. He does not become "locked in" on an operant that has been repeatedly reinforced, as the law of effects would predict. Instead, when the contingency for reinforcement is demanding, he ventures out to attempt other manipulanda, test out different strategies, often foregoing many reinforcements, until a more rewarding contingency is established. He acts like a creative scientist, testing hypotheses, formulating plans, and seeking ideal solutions. He does not passively accept "reality" as experienced; instead he seeks to create a "reality" as mapped in his blueprint. To him, the actualization of a blueprint is more reinforcing than the mere accumulation of more tangible reinforcements.
The acquisition of higher order operants demands more abilities than the acquisition of a simple first order operant. In the latter case, only one operant (Rop) is followed by a specified reinforcement $\left(\mathrm{S}^{R}\right)$ in the presence of some discriminative stimulus ( $\left.S^{D}\right)$. It involves the formation and retention of two simple associations: $\mathbf{S D}-\mathrm{Rop}$ and Rop-SR. In the case of higher order operant, the reinforcement is not a concrete event; its establishment depends on the organism's ability to count (number of responses and/or time required for the production of reinforcement for the first order operant); to retain, differentiate, and evaluate different contingencies. Further, the organism must also remember and differentiate various responses or strategies he has made before he can form the remote association between a particular hi-operant and the derived reinforcement. In short, a greater capability of retention, differentiation, and integration is necessary for the acquisition of hi-operants. Another contributing factor is an organism's ability to vary his behavior. A very limited response repertoire, or a strong tendency to perseverate in responses that have been proven unproductive would reduce the likelihood of successfully acquiring a positive hi-operant.

Apart from competence and ability, the acquisition of hi-operants is also closely related to motivational and personality variables. Once a first order operant has been acquired, some would stay with it in spite of the demanding or unsatisfactory contingency for reinforcement. However, there are always others who would prefer to venture into unknown territories in search of a better world. The search may be futile and costly, but it is from such risk-taking that progress is made.

In conclusion, it may be stated that at least two classes of operant behavior can be identified. In the case of first order operant, the environment (or the experimenter) decides on the contingency for reinforcement, and the organism is primarily passive and reactive. In the case of higher order operant, the organism controls and modifies existing reinforcement contingencies; he assumes a more active role, and acts in a way that can be considered as creative or intelligent. The often elaborate patterns of hi-operants are not the product of "shaping", but the creation of imagination and thinking. It is out of higher order operants that cultures and civilizations are born.

\section{NOTE}

1. Some of these results will be reported at the Psychonomic Society Meeting in Boston, November, 1974.

(Received for publication September 27, 1974.) 